Siegel, I believe (if I am mistaken, I apologize), has said that the next great step in mathematical progress will be the burning of all books on mathematics. Should that somewhat spectacular step be taken, let us hope that Dickson's Introduction and a few other works of mathematical art escape.

E. T. BeLL

\title{
THE NEW MECHANICS
}

Einführung in die Wellenmechanik. By Louis de Broglie. Translated into German by Rudolf Peierls. Leipzig, Akademische Verlagsgesellschaft M. B. H., 1929. iv +221 pp.

La Nouvelle Mécanique des Quanta. By George Birtwistle. Translated into French (and augmented by four appendices) by M. Ponte and Y. Rocard, with a preface by J. Hadamard. Paris, Albert Blanchard, 1929. vi +333 pp.

Gruppentheorie und Quantenmechanik. By Hermann Weyl. Leipzig, S. Hirzel, 1928. viii $+288 \mathrm{pp}$.

About two years ago the new wave mechanics acquired remarkable experimental support through the discovery of the hitherto quite unknown phenomenon of diffraction of electronic streams by crystals. This discovery affords a genuine counterpart to the earlier discovery of the photoelectric effect, for the first shows that heretofore one aspect of the nature of matter has been left out of consideration while from the second it appears that one aspect of the nature of light had long been overlooked. It is now generally recognized that the behavior of light is corpuscular as well as undulatory; the diffraction of an electronic stream compels us to recognize that the behavior of matter is undulatory as well as corpuscular.

The older quantum theory failed to meet the dilemma of this two-fold character of light and of course did not consider at all the corresponding duality in the nature of matter; and it found no explanation of the presence of half quantum numbers in the formulas of the Zeeman effect and band spectra. A new theory was therefore inevitable. The first step was taken by Louis de Broglie in his dissertation (1924). The wave mechanics initiated by de Broglie received remarkable development at the hands of E. Schrödinger. Another line of development is due to W. Heisenberg.

De Broglie's Wellenmechanik furnishes the best introduction to the new quantum mechanics which has come to the reviewer's attention. A reader who desires to begin with a more elementary exposition would probably do well to use the second edition of Materiewellen und Quantenmechanik by A. Haas (1929); in this book the simpler aspects of the new theories are presented in an illuminating way. The exposition by de Broglie would then serve to complete an introduction to these most remarkable developments of the newer physics.

The starting point of de Broglie's wave mechanics has its origin in his purpose to develop the theory in such a way that there shall be an intimate connection between the conception of a corpuscle and that of periodicity in 
phenomena so that the notion of the movement of the corpuscle shall be inseparable from that of wave propagation. How this intimate connection is brought about is best illustrated by the case of a single particle.

In order to present this example let us start from the Lorentz transformation of the restricted theory of relativity, taking this transformation in the form

$$
x_{0}=x, \quad y_{0}=y, \quad z_{0}=(z-v t) /\left(1-\beta^{2}\right)^{1 / 2}, \quad t_{0}=(t-\beta t / c) /\left(1-\beta^{2}\right)^{1 / 2},
$$

where the coordinates $\left(x_{0}, y_{0}, z_{0}, t_{0}\right)$ and $(x, y, z, t)$ refer respectively to a system at rest and a system with uniform velocity $v$ in the direction of the $z$-axis and where $\beta=v / c, c$ being the velocity of light. Let a particle be at rest in the system $\left(x_{0}, y_{0}, z_{0}, t_{0}\right)$. If waves are to be associated with such a particle it is natural to assume that they are stationary in this system, so that the time shall enter into their mathematical expression only in a factor of the form $\cos 2 \pi \nu_{0} t_{0}$, provided that the time origin has been suitably chosen. The constant $\nu_{0}$ is called the characteristic frequency of the particle. Let us now suppose that the same particle is observed from the system $(x, y, z, t)$. The form of the associated waves in this system may then be determined by means of the Lorentz transformation. Then the factor $\cos 2 \pi \nu_{0} t_{0}$ takes the new form $\cos 2 \pi \nu(t-z / \nu)$, where

$$
\nu=\nu_{0} /\left(1-\beta^{2}\right)^{1 / 2}, \quad V=c / \beta=c^{2} / v .
$$

Therefore, to an observer on the system $(x, y, z, t)$, the waves seem to have a frequency $\nu$ and to proceed with the phase velocity $V$. This result appears as a simple and direct consequence of the transformation characterizing the restricted theory of relativity.

It turns out that the group velocity of the waves, defined in the way indicated by Lord Rayleigh, is simply the ordinary velocity $v$ of the particle with reference to the system $(x, y, z, t)$.

Some additional hypothesis is necessary to bring the conception of associated waves into connection with the quantum theory; and this is made by supposing that the energy $W$ of the particle and its frequency $\nu$ are connected by the equation

$$
W=h \nu,
$$

where $h$ is Planck's constant.

Such, in its simplest aspect, is the starting point of the new wave mechanics of de Broglie. This is presented, together with some of its immediate consequences, in the third chapter (pp. 34-41) of de Broglie's Wellenmechanik. The earlier part of the book consists of an introduction (pp. 1-9) and two chapters devoted to some general preliminaries belonging to the classical mechanics both in its Newtonian and in its relativistic form. The introduction contains a highly interesting and (in the main) non-technical presentation of some of the leading ideas of the new mechanics.

From the simple starting point indicated in the third chapter the author proceeds in the fourth (pp. 42-58) to a general introductory investigation of wave propagation in the new mechanics and thence to a study of the differential equation of mechanical waves in the fifth chapter (pp. 58-67). Here one encounters the now classic differential equation of Schrödinger. In the 
sixth chapter (pp. 67-75) there is an account of the relations between classical mechanics and wave mechanics. The seventh chapter (pp. 75-87) is devoted to the principle of interference and the diffraction of electronic streams by crystals: it brings to attention what is perhaps the most remarkable experimental confirmation that the new wave mechanics has yet received. Some related matters are treated in the eighth chapter (pp. 87-94). The ninth chapter (pp. 95-105) is devoted to the simpler aspects of those questions of probability which are forced upon one's attention by the new mechanics and particularly by Heisenberg's principle of uncertainty (already brought before the reader in the fourth chapter). The tenth chapter (pp. 105-116) is devoted to the remarkable wave mechanics of light quanta: it is shown in particular that the Einstein theory of light quanta is an almost immediate consequence of the initial hypotheses of wave mechanics. The HeisenbergBohr theory is developed in the eleventh chapter (pp. 116-126), and the bearing of Heisenberg's principle of uncertainty on fundamental questions of experimental possibility is treated in the twelfth chapter (pp. 127-136), while the thirteenth (pp. 137-151) is devoted to a further investigation of wave propagation. Up to this point the author has confined his attention exclusively to the motion of a single particle in a given field. The remaining six chapters (pp. 152-219) treat of the wave mechanics of systems of particles, the general method of exposition being much like that employed in the earlier chapters for the simpler cases.

It is obvious that so brief a book can not contain a full treatment of the whole of the new quantum theory; but it probably serves the purpose of an introduction to the theory better than it would if it were more comprehensive. One who reads this book with understanding will be prepared to make effective use of the original memoirs.

De Broglie's conception of wave mechanics was set forth in his dissertation in 1924. In $1925 \mathrm{~W}$. Heisenberg presented his new quantum mechanics in its earliest form. Birtwistle, at the beginning of the opening chapter of his book named above, places this work of Heisenberg in the foreground of attention. He says: "The origin of the new quantum mechanics is found in a memoir by Werner Heisenberg which marks the beginning of a new epoch; it contains a new idea which has led to extraordinary developments in quantum mechanics during the last two years .... In the new mechanics, the equations have a form identical with that of the classical theory, but the variables do not obey the commutative law of multiplication; $\cdots$ the quantum conditions of the older theory are here replaced by equations for determining $x y-y x$; these equations contain Planck's constant $h . "$

After an introductory chapter on the origin and development of the new quantum mechanics, Birtwistle devotes six chapters to a discussion of various experimental facts and certain laws or principles which have been directly associated with them. He then introduces in the eighth chapter (pp. 63-70) the Heisenberg quantum mechanics of 1925 with its non-commutative multiplication, develops in the next chapter (pp. 71-78) the theory of Dirac, gives in the tenth chapter (pp. 79-84) Heisenberg's theory of the harmonic oscillator and continues for several chapters with related matters. The work of Schrödinger dominates Chapters XVII to XXII (pp. 139-189); its relation to 
the work of Heisenberg is brought out with care, especially in Chapter XX where the Heisenberg matrices are determined from the point of view of Schrödinger's theory. The theory of de Broglie is briefly treated in Chapter XXIII (pp. 191-200). At this stage the more important general ideas are before the reader. In the remainder of the book we continue to have experimental results and theoretical developments presented alternately or interpenetrating each other, as may be found convenient in exposition.

From this outline of Birtwistle's book it will be seen that his exposition is guided by considerations far removed from those which dominate de Broglie's. On account of this great difference the two volumes supplement each other. In Birtwistle's more attention is given to experimental results than in de Broglie's; and theoretical considerations are presented by the two authors in markedly different orders. The book by Weyl is very different in conception and treatment from each of the preceding. It resembles Weyl's Raum, Zeit, Materie in plan in that only half of it belongs to the author's field (mathematics) while the other half belongs to physics. The external circumstances leading to its preparation were as follows. In the winter semester of 1927$1928 \mathrm{Z}$ ürich was left without a theoretical physicist owing to the fact that Debye and Schrödinger were called elsewhere. Weyl sprang into the breach by transforming his announced lectures on group theory into lectures on group theory and quantum mechanics; and the book before us resulted from the systematic development of the material so organized. The exposition carries with it evidence of the two influences involved in its production. The theory of the representation of continuous groups is developed in such a way as to exhibit its close contact with quantum theory. "From this book, if it attains its goal," so speaks the author, "one should be able to learn the fundamentals of group theory and quantum mechanics; in the mathematical sections the physicist is kept chiefly in mind, in the physical sections the mathematician."

The book falls into five chapters: the first treats the basis of the unitary geometry; the second is devoted to quantum theory and contains what is necessary for an understanding of the ideas and methods of this theory; the third chapter develops the elementary parts of the theory of representations of groups; an application of this theory to quantum physics constitutes the fourth chapter. In these four chapters mathematics and physics alternate. In the fifth chapter, on the other hand, there is a combination of both mathematics and physics in that the theory of permutation groups and representations of them is developed and is applied to important problems in atomic physics.

The book is not well suited to the needs of a reader who would make his first excursion either into group theory or into the new quantum physics. But one who has already become acquainted with the fundamental aspects of these two disciplines will find here a stimulating exposition and will obtain fruitful ideas for continued meditation.

R. D. Carmichael 